\title{
Redaktionsgeschichte und Hermeneutik
}

\section{Die Frage der guten Werke in der Confessio Augustana}

Editionen geben nicht nur Texte wieder, sie schaffen auch kanonische Textformen. Dies gilt auch für die Ausgabe der Confessio Augustana im Rahmen der Neuausgabe der Bekenntnisschriften der Evangelisch-Lutherischen Kirche ${ }^{1}$. Nach dem frühen Tod von Gottfried Seebaß im Jahre 2008 wurde mir der Abschluss dieser Edition übertragen. Mit übernommen habe ich die maßgebliche Richtungsentscheidung, die Seebaß für diese Edition gefasst hatte: dass Leittext der Edition, lateinisch wie deutsch, die Editio princeps von 1531 sein sollte, nicht aber, wie es Günther Bornkamm sich noch für die Ausgabe von 1930 vorgenommen hatte, die „mit den heutigen Mitteln der Wissenschaft erreichbare ursprüngliche Gestalt“2 rekonstruiert werden sollte. ${ }^{3}$ In der nun wohl für einige Zeit maßgeblichen Edition liegt den Nutzerinnen und Nutzern also eine Textfassung vor, die weder der 1530 überreichten noch auch der 1580 in das Konkordienbuch eingegangen und jahrhundertelang rezipierten Fassung entspricht. Sie stellt vielmehr für den Text der CA die „von seinen Autoren selbst als gültige Fassung“ angesehene Form dar. ${ }^{4}$

Zum Gewicht dieser Entscheidung sei hier vermerkt, dass die Confessio $\mathrm{Au}$ gustana damit nach einem deutlich anderen Prinzip ediert worden ist, als es für die Edition des griechischen Neuen Testaments üblich ist: Diese folgt nach wie vor dem Ideal des 19. Jahrhunderts, einen Text zu rekonstruieren, der so nah wie möglich am Original ist. Der Unterschied zwischen norma normans und norma normata lässt sich an dieser Stelle nicht allein mit der allgemein gewachsenen editionsphilologischen Skepsis gegenüber solchen Rekonstruktionen begründen, sondern auch mit der theologischen Einsicht, dass die Ursprungsauthentizität für die Heilige Schrift selbst eine ganz andere Bedeutung hat als für die ohnehin ja auf Deutung eines anderen Textes ausgelegte Bekenntnisschrift.

1 Die Confessio Augustana, hg. zus. mit Gottfried Seebaß, in: Die Bekenntnisschriften der Evangelisch-Lutherischen Kirche. Vollständige Neuedition, hg.v. Irene Dingel, Göttingen 2014, 63-225 (im Folgenden BSELK); vgl. auch die beigegebenen Materialien, in: Die Confessio Augustana - Texte und Kontexte, in: Die Bekenntnisschriften der Evangelisch-Lutherischen Kirche. Quellen und Materialien, Bd. 1: Von den altkirchlichen Symbolen bis zu den Katechismen Martin Luthers, hg.v. Irene Dingel, Göttingen 2014, 35-218 (im Folgenden QuM).

2 BSLK, VII.

3 S. zu den Editionsprinzipien BSELK, 73f. (Leppin).

4 BSELK, 73 (Leppin).

Ә OpenAccess. (c) 2022 Volker Leppin, publiziert von De Gruyter. (cc) BY-NC-ND Dieses Werk ist lizenziert unter einer Creative Commons Namensnennung - Nicht kommerziell - Keine Bearbeitung 4.0 International Lizenz. https://doi.org/10.1515/9783110683868-010 
Insofern hielt und halte ich die für die Edition getroffene Entscheidung für berechtigt. Soll sie aber nicht zu neuen falschen Kanonisierungen führen, muss sie in einen hermeneutischen Kontext eingezeichnet werden, dessen Notwendigkeit ich im Folgenden anhand eines besonders markanten Beispiels nachvollziehen will. Insbesondere der deutsche Text dokumentiert zur Frage der guten Werke ein innertextliches Ringen, das im Folgenden zunächst wenigstens in Ansätzen offenzulegen ist, ehe eine hermeneutische Schlussüberlegung gewagt wird.

\section{Das textgenetische Problem}

Dass die Frage der guten Werke in der Entstehung der Confessio Augustana ein besonderes Problem darstellte, ist auf den ersten Blick offenkundig, schon allein dadurch, dass das Thema zweimal angegangen wird: in Artikel 6 und Artikel 20. Artikel $6^{5}$ behandelt die Frage recht knapp im unmittelbaren Anschluss an die Ausführungen über die Erlangung des Glaubens durch das Predigtamt im fünften Artikel. ${ }^{6}$ Der Artikel stellt fest, dass der Glaube Werke hervorbringen solle und man gute Werke tun müsse, betont dabei aber deren rein heilskonsekutive Stellung: zur Erlangung des Heils führt allein der „glauben umb Christus willen“7 . Hierzu beruft sich CA 6 auf ein Zitat aus dem Ambrosiaster: „Also ists beschlossen bey Gott, das, wer an Christum gleubt, selig sey und nicht durch werck, sondern allein durch glauben one verdienst vergebung der sunden habe.“8

Textgeschichtlich war dies zunächst die einzige geplante Aussage zu der Frage: Mehrere Manuskripte bezeugen, dass in der Vorbereitung der Confessio Augustana zunächst der jetzige Artikel 20 über die guten Werke noch Mitte Juni 1530 jedenfalls nicht einhellig vorgesehen war. ${ }^{9}$ Er hat demnach erst in den letzten

\footnotetext{
5 Vgl. zu diesem Artikel die leider sehr kurzen Ausführungen von Gerhard Müller/Vinzenz Pfnür, Rechtfertigung - Glaube - Werke, in: Hardnig Meyer/Heinz Schütte (Hg.), Confessio Augustana. Bekenntnis des einen Glaubens, Paderborn/Frankfurt a. M. 1980, 106-138, 122.

6 BSELK, 100.

7 BSELK, 100.

8 BSELK, 102.

$9 \mathrm{Na}$, die Nürnberger Übersetzung der lateinischen Fassung von Anfang Juni geht direkt vom 18. Artikel über die Sünde (entspricht später: Art. 19) zu Artikel 19 über beiderlei Gestalt des Sakraments (später: 22) über, kennt also noch weder Art. 20 über die guten Werke noch Art. 21 über die Heiligen. Nü 1, eine von dem Brandenburg-Ansbacher Kanzler Georg Vogler dem Kaiser am 16. Juni [QuM 79 (Leppin)] mit dem Hinweis: „So vbergeben wir Irer kay. Mt hiemit desselben ain lauter anzeigen Inn der eyl vfs kurzt gestellt“ (Urkundenbuch zu der Geschichte des Reichstages zu Augsburg im Jahre 1530, hg.v. Eduard Förstemann, Bd. 1: Von dem Ausgange des kaiserlichen
} 
zehn Tagen vor Überreichung seinen festen Platz in der CA bekommen, ${ }^{10}$ offenbar um ein empfundenes inhaltliches Defizit zu beheben - erst auf einer synchronen Lesestufe wird er zu einem „Kommentar“ zu CA 6, wie Otto Hermann Pesch es formulierte. ${ }^{11}$

Die mit der Frage verbundenen Schwierigkeiten zeichneten sich dabei schon in der handschriftlichen Bearbeitung von Art. 6 ab: Eine Ansbacher Zusammenstellung von Artikeln der CA aus Mitte Juni 1530 war bedeutend kürzer als die oben aus der Editio princeps referierte Fassung. In ihr fehlte nicht allein das Ambrosiaster-Zitat, sondern auch die Ausführungen zur bloß heilskonsekutiven Bedeutung der Werke sind deutlich knapper. ${ }^{12}$ Viel näher an der dann 1531 veröffentlichten Fassung ist aber bereits Mar, ein in Marburg aufbewahrtes Manuskript, das als „Handschrift mit hoher Nähe zum überreichten Text“, „möglicherweise [...] jenes Exemplar, das Philipp von Hessen oder seine Räte aus Augsburg in die Heimat gebracht haben“, gilt. ${ }^{13}$ Die Ergänzungen wurden also offenbar in den letzten zehn Tagen des Redaktionsprozesses vorgenommen parallel zu der Hinzufügung des kompletten Artikels 20.

\section{Confessio Augustana 20 in Texten rund um die Überreichung}

Für CA 20 gestaltet sich nun die Textgeschichte als so komplex, dass sie in der Neuedition nicht anders zu bewältigen war als durch einen Parallelabdruck der Editio princeps von 1531 mit der erwähnten Marburger Handschrift Mar aus dem unmittelbaren zeitlichen Umfeld der Überreichung. Eine Darstellung der Unterschiede im textgeschichtlichen, genauer: entstehungsgeschichtlichen Apparat hätte alles vernünftige Maß gesprengt. Eben deswegen soll nun auch zunächst der

Ausschreibens bis zu der Uebergabe der Augsburgischen Confession, Halle 1833, 280) vorgelegte Handschrift lässt unmittelbar auf Art. 19 Schlussformeln folgen, kennt also gleichfalls Art. 20 und 21 nicht.

10 Die von Spalatin erstellte deutschsprachige Fassung aus der Zeit Mitte Juni [s. QuM 75 (Leppin)] enthält Art. 20 bereits (Förstemann, Urkundenbuch, Bd. 1, 323-327), aber noch keinen Artikel von den Heiligen.

11 Otto Hermann Pesch, Rechtfertigung des Sünders und Gerechtigkeit in der Welt im Licht und Schatten des Augsburger Bekenntnisses, in: Bernhard Lohse/Otto Hermann Pesch (Hg.), Das Augsburger Bekenntnis von 1530 damals und heute, München u.a. 1980, 215-236, 220.

12 QuM, 81; vgl. BSELK, 100 App. zu ${ }^{\text {a-a }}$.

13 BSELK, 76 (Leppin): M30W; Zu den hier und im Folgenden verwendeten Kürzeln für Handschriften und Drucke s. BSELK, 74-83 (Leppin). 
Text von Mar untersucht und dargestellt werden, im folgenden Abschnitt dann die Editio princeps. Der Marburger Text steht dabei nicht allein. Ihm gehen mehrere Handschriften parallel. Hierzu gehört eine eigenhändige Handschrift Georg Spalatins von Mitte Juni 1530, ${ }^{14}$ eine Handschrift aus Brandenburg-Ansbachischem Besitz, die „recht nahe an das verschollene Übergabeexemplar bzw. in den bis zur Verlesung am 25. Juni 1630 reichenden Bearbeitungsprozess “ führt, ${ }^{15}$ eine Handschrift aus Coburgischem Besitz, ${ }^{16}$ eine Handschrift Nürnberger Provenienz, die „der endgültigen Fassung [...] schon recht nahe“ kommt, ${ }^{17}$ sowie eine im Archiv der Hansestadt Lübeck befindliche Handschrift, die mit einem Teil dieser Gruppe auch eine andere markante Gemeinsamkeit, nämlich die Einfügung Albrechts Grad von Mansfeld in der Unterschriftenliste, aufweist. ${ }^{18}$ Hinzu kommt noch ein 1530 bei Hans Walther in Magdeburg herausgebrachter Druck. ${ }^{19}$ Er gehört „zu einer Gruppe von Drucken, die zum Teil noch während des Augsburger Reichstages, jedenfalls im Jahr 1530, entgegen dem ausdrücklichen Publikationsverbot des Kaisers erschienen“20, die also eine Druckfassung auf dem Stand vor Erscheinen der Editio princeps repräsentieren. Diese Textzeugen weisen in CA 20 keine großen Abweichungen voneinander auf, unterscheiden sich aber gemeinsam markant von der Editio princeps. Wie die verschiedenen Hinweise auf ihren Entstehungszeitpunkt und -kontext deutlich machen, stehen sie dem Überreichungsexemplar nahe.

Das führt nun auf eine interessante Spannung zwischen den heute zu befolgenden philologischen Richtlinien einerseits, dem historisch weiterführenden Interesse andererseits. Philologisch könnte ein Text der überreichten CA nur unter Verwendung der üblichen, aber eben doch ungewissen textkritischen Hypothesen rekonstruiert werden. Einen solchen erstellten Text zu edieren, entspricht nicht mehr dem editionsphilologischen Hauptstrom. Und doch kann man in aller Vorsicht aus der Gruppe der in der Nähe der Überreichung entstandenen Handschriften eine Gestalt von CA 20, nicht ihrem Wortlaut, aber ihrem ganz überwiegenden Duktus nach, erschließen, die näher an das Reichstagsgeschehen führt als der dem Leithandschriften bzw. -druck folgende Text der Neuedition, der die deutlich spätere Fassung der Editio princeps wiedergibt. Die genannte Gruppe von Handschriften, unter welchen in der Edition wiederum Mar als Leithand-

\footnotetext{
14 QuM, 75 (Leppin): Weil.

15 QuM, 94 (Leppin): Nü2.

16 BSELK, 74 f. (Leppin): Co.

17 BSELK, 75 (Leppin): Ha.

18 BSELK, 76 (Leppin): Lü.

19 Beschreibung: BSELK, 77 f. (Leppin).

20 Beschreibung: BSELK, 77 (Leppin).
} 
schrift zur Darstellung der durchgängigen Parallele zur Editio princeps gewählt wurde, führt also näher an den reichsrechtlich relevanten Text als letztere, während diese, die Editio princeps, stärker das sich in Wittenberg ab 1531 formierende Selbstverständnis repräsentiert. Für die interkonfessionellen Lektüre bedeutet dies auch: Der Text der Handschriftengruppe führt näher an den deutschsprachigen Text, den die Confutatoren neben dem lateinischsprachigen kennen konnten.

Unter diesem Gesichtspunkt ist es nun also, ungeachtet der getroffenen editorischen Entscheidung, von hoher historischer und theologischer Relevanz, welchen Text die Handschriftengruppe zu Art. 20 bietet. Zunächst ist die apologetische Motivation der ausführlichen, über CA 6 hinausgehenden Behandlung des Themas deutlich: Der Artikel steigt mit der Bemerkung ein: „Den unsernn wirt mit unwarheit ufgelegt, das sie gute werck verpieten“21. Hatte also Art. 6 vornehmlich die rechte Zuordnung von Heilskonstitution und -konsekution behandelt, so ging es nun um den Vorwurf genereller Missachtung der Werke. Aus Perspektive Melanchthons und der anderen Autoren musste dies wie ein Schritt zurück erscheinen, da ja eigentlich Art. 6 schon klar eine positive Lehre über Werke entfaltete, hier diese aber aufs Neue im Grundsatz begründet werden musste. Das Problem also, das unter der in den meisten Handschriften beigegebenen Überschrift „Vom glauben und wercken“22 verhandelt wird, wird von letzterem Begriff, den Werken, und ihrer Legitimität aus gedacht. Beides aber, Glaube und Werke, wird dann verschränkt ineinander dargestellt. Diese wenig klare Gedankenführung resultiert möglicherweise auch daraus, dass die Autoren der CA sich nicht allein genötigt sahen, sich gegenüber dem Vorwurf, Werke zu verbieten, zu verteidigen, sondern sie zugleich dazu Stellung nehmen wollten, dass ihrer Wahrnehmung nach die Gegenseite begonnen habe, „nu vom glauben zurethn, davon sie doch in vortzeiten gar nichts gepredigt haben“23. Das bei den Gegnern beobachtete Motiv sei nun, dass sie von „glaub und werck“ sprächen. ${ }^{24}$ Aufgabe der CA-Autoren war es also, zugleich diese unterstellte positive Auffassung der Gegenseite des falschen Gebrauchs des Glaubensbegriffs zu überführen - und die eigene Auffassung als unschädlich für Werke zu erweisen. Und all dies musste, wie oben ausgeführt, binnen weniger Tage zu Papier gebracht werden.

Der doppelten Aussageabsicht entsprang es, dass CA 20 einen Abschnitt damit einleitete, man wolle nun erläutern, wie „underricht“ vom Glauben „be-

21 BSELK, 117a, 3-5.

22 BSELK, 117a, 2.

23 BSELK, 117a, 20 - 22.

24 BSELK, 117a, 27. 
scheen" sei, ${ }^{25}$ hier aber nicht etwa positive Ausführungen über den Glauben folgen ließ, sondern lediglich im Grundsatz die Ausführung aus CA 6 erneuerte, dass nicht die Werke, sondern allein der Glaube das Heil verdiente. ${ }^{26}$ Dieser Gedankengang diente der Verteidigung gegenüber dem Vorwurf, Werke zu verachten. Erst deutlich später erfolgte dann auch eine inhaltliche Bestimmung des Glaubens, deren Funktion nun klar ist, den zweiten Gedankenstrang aufzugreifen, nämlich die Verbindung von Glaube und Werke bei den Gegnern, und diesen zu zeigen, dass sie über einen unzureichenden Glaubensbegriff verfügten: $\mathrm{Zu}$ unterscheiden sei nämlich zwischen dem „historien glauben“, wie ihn auch der Teufel oder die Gottlosen hätten, und „warem glauben, der do glaubt, das wir durch Christum gnade und vergebung der sunde erlangen“"27. Dieser definitorische Einschub, der zugleich derjenige ist, an welchem die genannte Handschriftenfamilie die größte interne textkritische Abweichung aufweist, ${ }^{28}$ unterbricht die sehr viel längere, vielfach redundante Darlegung zur heilskonsekutiven Bedeutung der Werke. Expliziter als CA 6, wo der Bezug auf den Heiligen Geist durch den Anschluss an Art. 5 gewährleistet war, ${ }^{29}$ argumentiert CA 20 dabei pneumatologisch: Der Glaube vermittele den Heiligen Geist und dieser bewege das Herz zu guten Werken. ${ }^{30}$

Besonderes Augenmerk legen die Verfasser dabei auf den Beweis aus Schrift und Kirchenvätern. Traugott Holtz hat festgestellt, dass sich die Schriftzitate in der CA ab Art. 20 häufen und sich in den spänigen Artikeln steigern, und dies als Indiz dafür genommen, dass schon ab Art. 20 die Notwendigkeit der biblischen Legitimation in Auseinandersetzung mit der Gegenseite als besonders hoch angesehen wurde. ${ }^{31}$ So werden als Bibelstellen angeführt: Eph 2,8; ${ }^{32}$ Röm 5,1;33 Joh 15,5. Der interessanteste biblische Beleg ist dabei der Verweis auf Hebr 11,1: Er diente traditionell der erkenntnistheoretischen Reflexion, das heißt, der Bezug des Glaubens auf die Hoffnung wurde v.a. von der Erwähnung des nicht Sichtbaren

25 BSELK, 119a, $7 \mathrm{f}$.

26 BSELK, 119a, $10-22$.

27 BSELK, 123a, 25-29.

28 BSELK, 119a Anm. Zu r-r: Abweichung in Ha.

29 BSELK, 100, 3f.; vgl. Wilhelm Maurer, Historischer Kommentar zur Confessio Augustana, Bd. 2: Theologische Probleme, Gütersloh 1978, 151.

30 BSELK, 125a, $30-34$.

31 Traugott Holtz, Beobachtungen zu Umfang und Charakter der Schriftbegründung in der Confessio Augustana, in: Fritz Hoffmann/Ulrich Kühn (Hg.), Die Confessio Augustana im ökumenischen Gespräch, Berlin 1980, 73-85, 76.

32 BSELK, 119a, 29-121a, 1.

33 BSELK, 121a, 21-123a, 2. 
her gedeutet. ${ }^{34}$ Die CA betont nun die erste Vershälfte in material-theologischer Hinsicht, nach welcher Glaube eine feste Zuversicht der Hoffnung ist. ${ }^{35}$

Eben diese Deutung wird mit einem Verweis auf Augustin, der sich sachlich wohl auf den pseudo-augustinischen Liber de cognitione verae vitae beziehen lässt, unterstrichen. ${ }^{36}$ Ein Pauschalverweis aus Augustins De spiritu et littera wird ausdrücklich mit dem Vermerk eingeführt, daraus könne man beweisen, „das hierin kein neuer verstandt eingefurt sei“37. Diese Autoritätenverweise verbinden sich mit dem anthropologischen Argument, dass allein das Vertrauen auf Christus statt auf die eigenen Werke dem Gewissen Ruhe geben könne ${ }^{38}$ - genau dieser Glaube sei es, so die Aufnahme der definitorischen Bestimmungen, der in den vergangenen Jahrhunderten nicht gepredigt worden sei. ${ }^{39}$

Die Argumentationslinie von CA 20 im zeitlichen Zusammenhang der Überreichung des Bekenntnisses ist also bestimmt von der apologetischen Auseinandersetzung. Defensiv wird der Vorwurf abgewiesen, keine Werke zu lehren, offensiv wird der Gegenseite vorgeworfen, früher Werke ohne Glauben, nun Werke mit einem falschen, nämlich rein historischen Glaubensverständnis gelehrt zu haben. Das rechte Glaubensverständnis besteht in einem Ergreifen des Heilsgeschehens, welches den Heiligen Geist und so die Fähigkeit zu guten Werken vermittelt. Dem bei den Gegnern ausgemachten additiven Verständnis von Glaube und Werken als heilskonstitutiven Elementen ${ }^{40}$ wird eine Lehre von einem heilskonstitutiven Glauben, der die Werke aus sich heraussetzt, entgegengehalten.

\section{Confessio Augustana 20 in der Editio princeps}

Die in CA 20 mitgeführte Debatte mit der altgläubigen Seite wird erkennbar in der Confutatio, die sich ja auf die eingereichte Fassung, nicht auf die Editio princeps bezieht. Zu CA 6 widerspricht sie vor allem der alleinigen heilskonstitutiven Bedeutung des Glaubens ${ }^{41}$ - die argumentative Stoßrichtung liegt dabei umgekehrt

34 S. z. B. Thomas, Summa, II-II q. 4 a. 1 (Editio Leonina 8, 43f.).

35 BSELK, 125a, 13-17.

36 BSELK, 125a, 17-23.

37 BSELK, 121a, $1 \mathrm{f}$.

38 BSELK, 121a, $10-21$.

39 BSELK, 123a, 14-22.

40 BSELK, 117a, 27.

41 Die Confutatio der Confessio Augustana vom 3. August 1530, bearb. v. Herbert Immenkötter, Münster 1979 (CCath 3), 90, $18 \mathrm{f}$. 
zu der in CA 6: War hier betont worden, dass die Lehre von der Alleinigkeit des Glaubens Werke nicht verhinderte, hebt Conf 6 gemäß Jak 2,20 hervor, dass der Glaube ohne Werke tot sei. ${ }^{42}$ Im Blick auf Artikel CA 20 beobachten die Confutatoren recht genau dessen apologetischen Charakter: „Der zwenzigist artickel ist nit so seer der fursten und der stet bekantnus als ein entschuldigung irer prediger. “43 Inhaltlich rekurrieren sie schlicht auf ihre Ablehnung von CA 6, die ebenso auch für CA 20 gelte. ${ }^{44}$ Das ihnen vorgeworfene additive Verständnis der Zuordnung von Glauben und Werken führen sie dabei näher an die Lösung von CA 20 heran, indem sie betonen, dass die Werke nicht aus sich heraus, sondern ,allain aus krafft des verdinsts des leidens Christi“ verdienstlich seien. ${ }^{45}$ Hierfür berufen sie sich ihrerseits neben zahlreichen Bibelstellen auf Lehrverurteilungen „bey den zeiten Augustini“ ${ }^{“ 6}$, womit die Synode von Orange 529 gemeint sein dürfte. ${ }^{47}$ Die Trennlinie zwischen CA und Conf liegt also hiernach darin, ob die Werke zwar notwendig, aber bloß heilskonsekutiv sind (CA) - oder sekundär und partial heilskonstitutiv (Conf).

Melanchthon bot seinerseits zu CA 6 in ApolCA keine eigene Erklärung, sondern integrierte diese Frage in eine lange Erklärung zu CA 4, welche die beiden folgenden Artikel einschloss. Durch die Verweise auf die biblische Rede vom Lohn erklärte er hier, dass es nicht um Lohn zur Erlangung des Heils gehe, sondern zu anderen Gaben im jetzigen und künftigen Leben. ${ }^{48} \mathrm{Zu}$ CA 20 hob er hervor, dass eine Bestreitung der Lehre, dass die Vergebung der Sünde allein aus dem Glauben komme, einer Bestreitung der wichtigsten Grundlehre des Christentums überhaupt gleichkomme. ${ }^{49}$ Angesichts des kaiserlichen Edikts gegen die Evangelischen betonte Melanchthon, dass er an diesem Punkt gewiss sei, „das die widdersacher verdamnen die offentlichen, Göttlichen warheit und die rechten Christliche, selige, heilige lere, one wilche kein Christlich kirche irgent sein

42 Confutatio, $88,16 \mathrm{f}$.

43 Confutatio, 120, 12f. Zur Kritik der Confutatio an CA 6 und 20 s. Herbert Immenkötter, Um die Einheit im Glauben. Die Unionsverhandlungen des Augsburger Reichstages im August und September 1530, Münster 1973 (KLK 33), 18f.; Vinzenz Pfnür, Einig in der Rechtfertigungslehre? Die Rechtfertigungslehre der Confessio Augustana (1530) und die Stellungnahme der katholischen Kontroverstheologie zwischen 1530 und 1535, Wiesbaden 1970 (VIEG 60), 237-240, 244-250.

44 Confutatio, 120, $15 f$.

45 Confutatio, 122, 13-15.

46 Confutatio, 122, $26 \mathrm{f}$.

47 Synode von Orange c. 18: Nullis meritis gratiam praeveniri. Debetur merces bonis operibus, si fiant; sed gratia, quae non debetur, praecedit, ut fiant (DH 388). Es handelt sich hierbei um ein Zitat aus Augustin, Opus imperfectum contra Iulianum I, 133 (PL 45, 1133).

48 BSELK, 392, 1-3.

49 BSELK, 554, 2-13. 
kann“50 . Art. 20, der erst in der letzten Phase in die CA gekommen war, erhielt so also, wohl aufgrund der Einsicht in den engen Zusammenhang mit dem Artikel 4 von der Rechtfertigung, eine eminente Zentralstellung innerhalb des Gesamtgefüges. Dies dürfte auch den Hintergrund dafür bilden, dass Melanchthon hier und eben in Art. $4^{51}$ - einschneidenden Änderungsbedarf sah: Die reformatorische Grundaussage bedurfte der Klärung.

Diese Klärung erfolgte auf mehrere Weisen. Der auf den ersten Blick auffälligste Schritt ist, dass der Art. 20 der Confessio Augustana in der Editio princeps als einziger unter allen Artikeln eine Binnengliederung erfuhr. Er ist nun eingeteilt in einen Abschnitt „Wo glaube und was der glaube sey“52 und einen „Das man gute werck sol und müsse thun und wie man sie könne thun und wie sie Gott gefellig sein" ${ }^{53}$. In dogmatischer Perspektive sind nun also die Fragen von Glaube und Werk je einzelnen Abschnitten zugewiesen. In genetischer Perspektive wird man sagen können, dass die unterschiedlichen Aspekte apologetischer Ausrichtung, die den Überreichungstext des Reichstages prägten, nun in je eigene Abschnitte gesetzt wurden. Direkt wird die apologetische Ausrichtung nur noch im allerersten Abschnitt des Artikels, der weitgehend an die vorherigen Fassungen anknüpft, benannt. ${ }^{54}$

Bedeutsamer als diese äußerliche Änderung ist aber die inhaltliche, die sich durch den gesamten Artikel zieht und auch in der parallelen Bearbeitung von CA 4 erkennbar ist: So wie in diesen gegenüber den verschiedenen greifbaren vorherigen Fassungen die Lehre von „Gottes gesetz“ und „der verheissung Christi“ eingezeichnet wurde, ${ }^{55}$ ist nun auch Art. 20 klarer schrifttheologisch durchstrukturiert. Die Bestimmung des Glaubens beginnt mit einer kurz zusammengefassten Lehre von der Predigt der Buße und der Vergebung durch das „Evan-

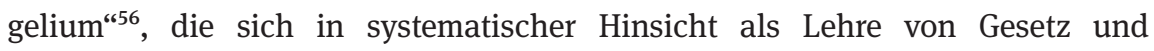
Evangelium fassen lässt. Streng durchdacht, wird nun auch in die Definition des Glaubens bereits die Abwehr gegenüber dem auf moralische Laxheit hinauslaufenden Vorwurf, die Werke zu beseitigen, hineingenommen: „hieraus ist auch zu mercken, wo glauben sey und was wir glauben heissen. Denn wo nicht schrecken ist fur Gottes zorn, sondern lust $\mathrm{n}$ sündlichem wandel, da ist nicht glauben, denn

50 BSELK, 556, 12-15.

51 S. die Synopse der ebenso komplexen Überlieferungslage in BSELK, $98 \mathrm{f}$.

52 BSELK, 116, 18.

53 BSELK, 120, $31 \mathrm{f}$.

54 BSELK, 116, 3-17.

55 BSELK, 98, 7. 20.

56 BSELK, 98, 19-25. 
glauben sol trösten und lebendig machen die erschrockne hertzen. "57 Indem in die Glaubensdefinition der Schrecken über die Sünde integriert wird, ist deutlich, dass die Rechtfertigungslehre gerade nicht als Freibrief zum Sündigen zu verstehen ist ${ }^{58}$ - erst von hier aus kommt die Neufassung dann auf den bloß historischen und damit nicht heilbringenden Glauben des Teufels zu sprechen. ${ }^{59} \mathrm{Im}$ zweiten Abschnitt wird die pneumatologische Wirkung des Glaubens präzisiert, die hiernach nicht allein im Wirken der Werke besteht, sondern gleichermaßen in Erkenntnis der Sünde und Glauben an die Zusage der Barmherzigkeit. ${ }^{60}$ Erst darauf folgt der Hinweis auf die Hilfe des Geistes zur Gebotserfüllung. ${ }^{61}$

Diese Formulierungen sind erkennbar nicht mehr von der apologetischen Haltung gegenüber den Gegnern getrieben. Sie zeigen vielmehr das Bemühen um eine schlüssige, dogmatisch ausgereifte Ausformulierung des Verhältnisses von Glauben und Werken. Insofern dokumentieren sie in gewisser Weise bereits 1530/ 31 das, was Wolf-Dieter Hauschild als Funktionswandel der Confessio Augustana „von einem Konsensuspapier zur ,Gründungsurkunde“ der lutherischen Kirche“ beschrieben hat. ${ }^{62}$ Der nachgezeichnete Entwicklungsprozess wirft aber auch hermeneutische Fragen auf.

\section{Hermeneutische Schlussüberlegungen}

Die vorliegenden Darlegungen haben einen erheblichen textlichen und theologischen Variationsprozess nachgezeichnet. Die theologische Grundsatzproblematik der Stellung der guten Werke im evangelischen Glauben schlägt sich in einem Geflecht zahlreicher intertextueller Bezüge zwischen Texten nieder, die von sich jeweils behaupten, der gerade zu diesem Zeitpunkt vorhandene Text der Confessio Augustana, sei es auf Entwurfs-, sei es auf Rezeptionsstufe zu sein. Intertextualität und Textidentität liegen also unentwirrbar ineinander.

Die Editio princeps der Confessio Augustana präsentiert aus diesem Ringen einen Text, dem man einerseits gewachsene theologische Durchdringung attes-

57 BSELK, 120, $10-13$.

58 Vgl. BSELK, 120, 14-16.

59 BSELK, 120, $19-22$.

60 BSELK, 122, 2-5.

61 BSELK, 122, 5-9.

62 Wolf-Dieter Hauschild, Das Selbstverständnis der Confessio Augustana und ihre kirchliche Relevanz im deutschen Protestantismus, in: Karl Lehmann/Edmund Schlink (Hg.), Evangelium Sakramente - Amt und die Einheit der Kirche. Die ökumenische Tragweite der Confessio Augustana, Freiburg i.B./Göttingen 1982 (DialKir 2), 133-163, 133. 
tieren mag, andererseits aber auch, damit verbunden, ein höheres Maß an Exklusivität gegenüber dem altgläubigen Gegenüber - daher gibt ihn auch die Neuedition nur als eine Variante neben anderen wieder, zwischen denen auf editorischer Ebene keine normative Entscheidung möglich ist. Dieser Befund ist, bedenkt man die unterschiedlichen Situationen der Übergabe in Augsburg am 25. Juni 1530 einerseits, der Drucklegung nach ausgebliebener reichsrechtlicher Billigung des Textes andererseits, nicht erstaunlich. Er bedarf aber im ökumenischen Horizont der hermeneutischen Reflexion.

Den Text von 1531 als den normativen Text der Confessio Augustana schlechthin zu nehmen, wäre nicht nur historisch, sondern auch theologisch falsch. Historisch wäre dies falsch, weil es sich hierbei weder um den reichsrechtlich relevanten (und mit historischen Mitteln nicht zweifelsfrei rekonstruierbaren) 1530 überreichten noch um den seit 1580 rezipierten Text handelt, der seinerseits textkritisch sogar eine Rückkehr zu einer den rund um die Übereichung nachweisbaren Überlieferungen nahestehenden Fassung darstellt. ${ }^{63} \mathrm{Er}$ repräsentiert einen bedeutsamen Ausschnitt aus der Textgeschichte der CA, mehr auch nicht - und dieser Ausschnitt fällt ausgerechnet in die Zeit, bevor, nach Hauschild etwa ab 1534/35, die CA als ,generelle Lehrnorm, an welche sich die Pfarrer zu halten hätten“, thematisiert wurde. ${ }^{64}$ Grundsätzlich sind alle Fassungen der CA, jedenfalls alle, denen auf unterschiedlichen Ebenen des Entscheidungsprozesses - bei der Verlesung 1530, bei der Veröffentlichung der Editio princeps wie bei der Herausgabe des Konkordienbuches - Gültigkeit zugesprochen wurde, als unterschiedlich formulierte definitive Bestimmungen evangelischer Lehre anzusehen. Die Formulierungsvielfalt provoziert dann, auf den zugrunde liegenden inhaltlichen Grund und Gegenstand zu schauen.

Die Edition legt - nicht zuletzt auch dadurch, dass sie offensiv die unterschiedlichen Varianten präsentiert - offen, dass die Rede von „der“ Confessio Augustana im Singular eigentlich ein Konstrukt ist. Ja, Melanchthon selbst hat streng genommen durch die Fassung von 1531 gerade nicht einen Text vorgelegt, den er „selbst als gültige Fassung angesehen“ hätte, ${ }^{65}$ sondern er hat durch den 1533 einsetzenden Bearbeitungsprozess und die Produktion einer ganzen Anzahl von variata-Fassungen ${ }^{66}$ deutlich gemacht, dass es einen festen Text der CA gerade nicht gibt. Man könnte zugespitzt sagen, dass die jetzige Edition die CA so präsentiert, wie sie von 1531 bis 1533 Gültigkeit besaß. Eben dies gibt im Umgang

63 BSELK, 73 (Leppin).

64 Wolf-Dieter Hauschild, Relevanz, 148; Hauschild erwägt ebd. bei entsprechender Datierung des Wittenberger Doktoreides auch einen Beginn ab 1533.

65 So meine eigene, entschieden korrekturbedürftige Formulierung BSELK, 73 (Leppin).

66 S. hierzu BSELK, 72 (Leppin); QuM 108-218. 
mit dem Text Freiheit, ohne dass diese in Bindungslosigkeit umschlagen müsste: Die verschiedenen Fassungen der Confessio Augustana erweisen sich als unter verschiedenen Perspektiven erfolgte Reformulierungen theologischer Kerngehalte. Wo 1530 das Werben um die altgläubige Seite im Vordergrund stand, ging es 1531 um die Selbstverständigung im Wittenberger Lager, 1540 aber um die Einigung mit den Oberdeutschen. Das ist nicht Ausdruck von Beliebigkeit, sondern gibt wieder, dass die unverzichtbaren Kerninhalte evangelischen Glaubens unter unterschiedlichen Bedingungen bis in den Bekenntnistext hinein unterschiedlicher Formulierungen bedürfen. Die Redaktionsgeschichte der Confessio Augustana mag damit Mut machen, sich in Verantwortung gegenüber der Heiligen Schrift und dem Bekenntnis in seinen unterschiedlichen Formulierungen, neu zu fragen, welches im ökumenischen Horizont der Gegenwart der unverzichtbare Grund und Gegenstand des Glaubens ist - und sich nicht zu scheuen, darin ein hohes Maß an Gemeinsamkeit mit den römisch-katholischen Geschwistern zu entdecken. 